Supporting Information:

\title{
Atomic-Scale Hidden Point-Defect Complexes Induce Ultrahigh Irradiation Hardening in Tungsten
}

\author{
Ruo-Yao Zheng ${ }^{1}$, Wu-Rong Jian², Irene J. Beyerlein ${ }^{2,3}$, Wei-Zhong Han ${ }^{1 *}$ \\ ${ }^{1}$ Center for Advancing Materials Performance from the Nanoscale, State Key Laboratory for \\ Mechanical Behavior of Materials, Xi'an Jiaotong University, Xi'an 710049, China; \\ ${ }^{2}$ Department of Mechanical Engineering, University of California, Santa Barbara, CA 93106-5070, \\ USA \\ ${ }^{3}$ Materials Department, University of California, Santa Barbara, CA 93106-5070, USA
}

Experimental and calculation methods

Figures S1-S4

Table S1

Movies S1 and S2 


\section{Experimental methods}

\section{Helium implantation and defects distribution after irradiation}

Tungsten single crystal foils with dimensions $1.5 \mathrm{~mm}$ (width) $\times 2 \mathrm{~mm}$ (length) $\times 40 \mu \mathrm{m}$ (thickness) were used. In order to fabricate samples with different He concentrations, tungsten foils were irradiated using $400 \mathrm{keV} \mathrm{He}$ ions at $400^{\circ} \mathrm{C}$ to a dose of $2 \times 10^{17}$ ions $/ \mathrm{cm}^{2}$ and $5 \times 10^{17}$ ions $/ \mathrm{cm}^{2}$, respectively. The variation of radiation damage (the blue curve, displacement per atom-dpa) and He concentration (the red curve, at.\%) with depth are plotted in Fig. S1a. Radiation damage (the blue curve, displacement per atom-dpa) and helium concentration (the red curve, at.\%) along depth were estimated using the Stopping and Range of Ions in Matter (SRIM) simulation, ${ }^{1}$ using the full cascade damage mode and a threshold displacement energy of $90 \mathrm{eV}$ and lattice binding energy of $0 \mathrm{eV}$ for tungsten. ${ }^{2,3}$ The simulated He concentration matches well with the distribution of detectable He bubbles, as shown in Figs. S1 and S2. Dense He bubbles with diameters in the range of 1-2 $\mathrm{nm}$ were produced at depths ranging from 400 to $1000 \mathrm{~nm}$.

We selected three areas--two within the irradiated region and one in an unirradiated region--within each sample for compression testing. The first type of sample has a low He concentration (about 0.7 at.\%) at depths of $200-400 \mathrm{~nm}$. Dislocation loops and a few He bubbles are observed in this region, as shown in Fig. S1b. The second type of sample has a higher He concentration (9 at.\%) for depths of $600-800 \mathrm{~nm}$. Large densities of He bubbles dominate this region, as shown in Figure S1c. The unirradiated samples were fabricated from a region lying 10 $\mu \mathrm{m}$ beyond the implanted region. These samples are completely free of radiation defects, as shown in Figure S1d. The defect distribution and range of depths associated with these three regions from a sample irradiated with a fluence of $2 \times 10^{17}$ ions $/ \mathrm{cm}^{2}$ are similar to the three regions taken from a sample irradiated with a fluence of $5 \times 10^{17}$ ions $/ \mathrm{cm}^{2}$. For the latter sample, the He concentrations are, of course, much higher, such as 1.9 at.\% at the depth of $200-400 \mathrm{~nm}$ and 20 at.\% at the depth of $600-800 \mathrm{~nm}$. For simplicity, these pillars were named as $\mathrm{W}-0, \mathrm{~W}-0.7$, W-1.9, W-9 and W-20 according to their He concentrations.

\section{In situ compression test}

Focused ion beam (FIB, FEI Helios Nanolab 600) was utilized to fabricate a series of 
rectangular single crystal tungsten pillars with side length $D$, i.e., $D=\sqrt{A}$ (where $A$ is the cross-section area of the pillar) equal to about 100 to $150 \mathrm{~nm}$. The size effect can be reduced through cutting pillars with similar geometry and dimension. An accelerating voltage of $30 \mathrm{kV}$ and an ion beam current of $9.7 \mathrm{pA}$ were used for final machining. Pillars were machined at different depths in the irradiated tungsten in order to tune the He concentration. For example, pillars with He concentrations of 0.7 at. $\%\left(2 \times 10^{17}\right.$ ions $\left./ \mathrm{cm}^{2}\right)$ and 1.9 at. $\%\left(5 \times 10^{17}\right.$ ions $\left./ \mathrm{cm}^{2}\right)$ were cut at depths of 200-400 nm. Pillars with higher He concentrations were cut at depths of 600-800 nm corresponding to 9 at.\% and 20 at.\% for the two fluences. Unirradiated tungsten pillars were cut in regions far from the implanted range (depths $>10 \mu \mathrm{m}$ ). The $\mathrm{W}-0, \mathrm{~W}-9$ and $\mathrm{W}-20$ pillars were compressed along the [411] loading axis, and the W-0.7 and W-1.9 pillars were loaded along the [200] axis.

In situ compression tests were conducted using a Hysitron Picolndenter (PI95) inside a JEOL $2100 \mathrm{~F}$ transmission electron microscope with accelerated voltage of $200 \mathrm{keV}$. In-situ nano-mechanical tests were carried out under the displacement control mode with the strain rate programmed to be $5 \times 10^{-3} / \mathrm{s}$ and the displacement rate is $\sim 1.5 \mathrm{~nm} / \mathrm{s}$ for compression (strain rate of $5 \times 10^{-3} / \mathrm{s}$ ). Prior to testing, pillars were carefully aligned with the diamond punch to ensure uniaxial compression. During compression, the load and displacement data can be recorded. The entire evolution of specimen deformation during loading was captured by a Charge-Coupled Device (CCD, Gatan 833) with 10 frames per second. Morphologies of W-0, W-0.7, W-1.9, W-9 and W-20 after compression are seen in Figure S3. Compression on thin foil tungsten pillars with and without radiation defects were also performed with a strain rate of $5 \times 10^{-3} / \mathrm{s}$. All irradiated samples were recorded with a defocus of $-1000 \mathrm{~nm}$ to monitor the evolution of the He bubbles. High resolution deformation tests of the $\mathrm{W}-0$ and $\mathrm{W}-20$ pillars are shown in Movies S1 and S2. Both samples were compressed along the [31ㄹ] axis and viewed along the [111] axis.

\section{Estimation of irradiation hardening}

Three commonly used irradiation hardening models were used to estimate the amount of hardening induced by the irradiation defects. ${ }^{1-3}$ The size, density, spacing and pressure of the He bubbles are key factors governing the level of hardening. Bubble size and density were counted 
by Image Pro Plus based on a series of TEM images. Bubble spacing was calculated by $\mathrm{l}=$ $1 / \sqrt{N d}$, where $N$ is bubble density and $d$ is bubble diameter. ${ }^{4}$ The bubble pressure was estimated by $P=4 \gamma / d$, where $\gamma$ is the surface energy of tungsten. ${ }^{4}$ Details on the properties of the He bubbles in the four types of samples are shown in Table S1. In the following, we estimated the helium bubble hardening using three popular analytical models with different assumptions on the nature (shearable or shear-resistant) of helium bubbles.

According to the Bacon-Orowan formula ${ }^{4}$, the hardening is given as

$$
\Delta \tau=\frac{\mu b}{2 \pi l}\left[\ln \left(d^{-1}+l^{-1}\right)^{-1}+\Delta\right]
$$

where $\Delta \tau$ is the increase in critical resolved shear stress by bubble hardening alone, $\mu$ is the effective shear modulus (161 GPa for W), $b$ is Burgers vector ( $0.274 \mathrm{~nm}$ for $\mathrm{W}$ ), $d$ is bubble diameter $(1.7 \mathrm{~nm}$ for $\mathrm{W}-0.7,17 \mathrm{~nm}$ for $\mathrm{W}-1.9,1.44 \mathrm{~nm}$ for $\mathrm{W}-9$ and $1.49 \mathrm{~nm}$ for $\mathrm{W}-20), l$ is bubble spacing (205 nm for W-0.7, $192 \mathrm{~nm}$ for W-1.9, $21.32 \mathrm{~nm}$ for W-9 and $17.47 \mathrm{~nm}$ for W-20) and $\Delta$ is a constant correlated to the penetrability of the obstacle. For a bubble, we set $\Delta$ to 0.77 , assuming that it can be cut by the moving dislocation. This model assumes a random distribution of obstacles and takes into account the interaction between the two dislocation segments as they bow around the obstacle. Hardening values $\Delta \tau$ for the five irradiated samples based on the Bacon-Orowan model are plotted in Fig. 1b with orange columns.

We consider also the Friedel model ${ }^{5}$ to provide another estimate for hardening distinct from Eqn (1), which is expressed as,

$$
\Delta \tau=\frac{\mu b}{2 \pi l} \ln \left(\frac{l}{r \cos \varphi}\right)(\cos \varphi)^{\frac{3}{2}}
$$

where $r$ is bubble radius, $\varphi$ is half the critical bow-out angle between lines of a dislocation when shearing the obstacle $\left(\cos \varphi=\frac{\ln (a D / b)}{\ln (l / b)}\right.$ and $\left.\frac{1}{D}=\frac{1}{d}+\frac{1}{l}\right)$, and the other parameters have the same meaning as explained for Eqn. (1). The increase in shear stress $\Delta \tau$ according to Friedel is displayed in Fig. $1 \mathrm{~b}$ by the green columns.

The third model is the dispersed barrier hardening (DBH) model $^{6}$ for impenetrable obstacles, unlike the prior models, and is given by 


$$
\Delta \tau=\alpha \mu b \sqrt{N d}
$$

where $N$ is bubble density $\left(1.4 \times 10^{22} / \mathrm{m}^{3}\right.$ for $\mathrm{W}-0.7,1.6 \times 10^{22} / \mathrm{m}^{3}$ for $\mathrm{W}-1.9,1.53 \times 10^{24} / \mathrm{m}^{3}$ for $\mathrm{W}-9$ and $2.2 \times 10^{24} / \mathrm{m}^{3}$ for $\mathrm{W}-20$ ) and $\alpha$ is typically referred to as a barrier strength coefficient, and commonly takes on a value of 0.2 for He bubbles with size less than $2 \mathrm{~nm},{ }^{6}$ which we used in the present calculation. Other parameters have the same meaning as those in Eqn. (1). The increased shear stresses $\Delta \tau$ by DBH are shown in Fig. $1 \mathrm{~b}$ with purple columns.

\section{Estimation of helium atoms in helium bubbles}

The percentage of He atoms in He bubbles were calculated by one of three methods. ${ }^{4,5,8-10}$ The first method is based on the bubble pressure ${ }^{5}$ :

$$
\begin{gathered}
P=\frac{2 \gamma}{r}+\sigma \\
\sigma=\frac{\mu b}{r}
\end{gathered}
$$

where $P$ is bubble pressure, $\gamma$ is the surface energy, $r$ is the bubble radius, $\sigma$ is the stress around the bubble, $\mu$ is the shear modulus, and $b$ is the Burgers vector. Two bounds of bubble pressure are obtained when $\sigma=0$ and $\sigma=\mu b / r$, from which the corresponding He atomic density $\rho$ can be referred ${ }^{8}$. If we assume that the He density is the average of these two bounds, then the number of He atoms in each bubble can be estimated as

$$
N_{H e}=\frac{4 \pi r^{3} \rho}{3}
$$

In our experiments, the number density of He bubbles $\rho_{b}$ can be counted by Image Pro Plus. The total number of helium atoms in all helium bubbles is related to $\rho_{b}$ via

$$
N=N_{H e} \rho_{b}
$$

The implanted He atoms can be calculated by SRIM:

$$
N_{\text {implant }}=N_{S} F
$$

where $N_{S}$ is the implanted $\mathrm{He}$ ions at certain depth and $F$ is radiation dose. Combining Eqns. (7) and (8), the percentage of $\mathrm{He}$ atoms in the bubbles is computed as

$$
f=\frac{N}{N_{\text {implant }}}
$$

The number of vacancies in each bubble can be estimated as

$$
N_{v}=V_{b} N_{w}
$$


where $V_{b}$ is the volume of one bubble, and $N_{w}$ is the atomic density of W. Then, the He/V ratio was calculated by $N_{H e} / N_{V}$. According to this method, the percentages of He atoms in the bubbles of $\mathrm{W}-0.7, \mathrm{~W}-9, \mathrm{~W}-1.9$ and $\mathrm{W}-20$ are $2.5 \%, 9.6 \%, 1 \%$ and $5.9 \%$, respectively.

The second model is based on the following formulation for bubble pressure and number of He atoms per bubble, ${ }^{4,9}$

$$
\begin{gathered}
P=\frac{2 \gamma+\mu b}{r} \\
V=\left(22.575+0.0064655 T-7.2645 T^{-\frac{1}{2}}\right) P^{-\frac{1}{3}}+(-12.483-0.024549 T) P^{-\frac{2}{3}}+ \\
\left(1.0596+0.10604 T-19.641 T^{-\frac{1}{2}}+189.84 T^{-1}\right) P^{-1} \\
N_{H e}=\frac{V_{b}}{V}
\end{gathered}
$$

where $V$ is the molar volume, $T$ is the absolute temperature, and other parameters have the same meaning as those of the first method. Calculation of the percentage of He atoms in the bubbles and $\mathrm{He} / \mathrm{V}$ ratio are the same as that in the first method. Consequently, the proportion of He atoms in the He bubbles calculated by the second method are $3.5 \%, 13 \%, 1.6 \%$ and $8.2 \%$ for W-0.7, W-9, W-1.9 and W-20, respectively, which are higher than those by the first method.

The third method was introduced according to following, ${ }^{10}$

$$
\begin{gathered}
P=\frac{2 \gamma}{r} \\
\frac{P}{K_{b} T n_{p}}=Z \\
C_{H e}=\frac{4 r^{3} \pi n_{p} \rho_{b}}{3 N_{W}} 10^{6}
\end{gathered}
$$

where $K_{b}$ is the Boltzmann constant $\left(1.38 \times 10^{-23} \mathrm{~J} / \mathrm{K} /\right.$ atom $), n_{p}$ is the helium density in bubble; $Z$ is the compressibility factor, $C_{H e}$ is He concentration and $N_{W}$ is the atomic density of tungsten. The percentage of $\mathrm{He}$ atom in bubbles can be estimated as

$$
f=\frac{C_{H e}}{C}
$$

where $C$ is the $\mathrm{He}$ concentration at certain areas. In our experiments, the percentage of $\mathrm{He}$ atom in bubbles for $\mathrm{W}-0.7, \mathrm{~W}-9, \mathrm{~W}-1.9$ and $\mathrm{W}-20$ are $2 \%, 11 \%, 0.8 \%$ and $9 \%$, respectively.

All three methods show similar results for percentage of He in those TEM-visible bubbles 
and the maximum value is $13 \%$, as listed in Table 1 and plotted in Fig. 4 a.

\section{Molecular dynamic simulations}

Molecular dynamics (MD) simulations are conducted using Large-scale Atomic/Molecular Massively Parallel Simulator (LAMMPS). ${ }^{11}$ To represent the atomic interactions of W-W, W-He and He-He, we choose the embedded-atom-method (EAM) potentials developed by Bonny et al. ${ }^{12}$. This potential has been successfully applied previously to study the tensile behaviors of $\mathrm{W}$ containing the embedded $\mathrm{He}$ atoms ${ }^{13}$ and the interactions between the dislocation and He atoms. ${ }^{14,15}$

Fig. S4 presents a schematic of the model set up with an initially straight dislocation and He/vacancy complex on the same plane embedded in a W single crystal. In the model, the [111], $[1 \overline{2}]$ and [110] crystallographic orientations are aligned along $x-, y$ - and $z$-axes, respectively. An edge dislocation or mixed dislocation with the character angle of $19.47^{\circ}$ and line orientation along $[11 \overline{2}]$ is then inserted on the middle (110) glide plane. Next, a He/vacancy complex is created on the right side of the dislocation along the $\mathrm{x}$ direction on the same dislocation glide plane. He/vacancy complexes are constructed with different He-atom to $\mathrm{n}$ vacancy ratios, denoted by $\mathrm{He}_{n} \mathrm{~V}(\mathrm{n}=1,2,3,4,5$ and 6). Periodic boundary conditions (BCs) are imposed along the $\mathrm{x}$ and $\mathrm{y}$ directions, while a non-periodic $\mathrm{BC}$ is applied along the $\mathrm{z}$ direction. The $\mathrm{x}$ and $\mathrm{z}$ dimensions for the whole system are 40 and $50 \mathrm{~nm}$, respectively. Their ratio of 0.8 is set to minimize any influences of image stress while the dislocation interacts with the obstacle. ${ }^{16}$

After constructing the model, dynamic relaxation at $300 \mathrm{~K}$ is conducted with NPT ensemble applied. During this thermostat process, two vacuum regions above the top surface and below the bottom surface along z-axis are created and maintained for 150 ps until the end of this relaxation step. For shear loading, we divide the whole model into three parts along the $z$ direction: the boundary regions, the thermostat regions and the non-thermostat regions, as shown by the red, green and non-colored regions, respectively, in Fig. S4. The thicknesses of the boundary regions, the thermostat regions and the non-thermostat regions are set to $1 \mathrm{~nm}, 10 \mathrm{~nm}$ and $28 \mathrm{~nm}$, respectively. The NVT and NVE ensembles are used for the thermostat and non-thermostat regions to avoid any artificial friction that could arise from the thermostat operation on dislocation mobility. By using the method of flexible $\mathrm{BC}^{11}$ we impose a constant 
velocity of $v$ to the top boundary region and maintain the bottom boundary static, which gives rise to a constant shear strain rate of $10^{7} / \mathrm{s}$.

\section{REFERENCES}

1 Ziegler, J. F., Ziegler, M. D. \& Biersack, J. P. SRIM - the stopping and range of ions in matter. Nucl. Instrum. Methods Phys. Res. B 268, 1818-1823 (2010).

2 Xu, Q., Yoshiie, T. \& Huang, H. C. Molecular dynamics simulation of vacancy diffusion in tungsten induced by irradiation. Nucl. Instrum. Methods Phys. Res. B 206, 123-126 (2003).

3 Stoller, R. E., Toloczko, M. B., Was, G. S., Certain, A.G., Dwaraknath, S. \& Garner F. A. On the use of SRIM for computing radiation damage exposure. Nucl. Instrum. Methods Phys. Res. B 310, $75-80$ (2013).

$4 \quad \mathrm{Li}, \mathrm{N} .$, Nastasi, M. \& Misra, A. Defect structures and hardening mechanisms in high dose helium ion implanted $\mathrm{Cu}$ and $\mathrm{Cu} / \mathrm{Nb}$ multilayer thin films. Int. J. Plast. 32-33, 1-16 (2012).

5 Wei, Q. M., Li, N., Mara, N., Nastasi, M. \& Misra, A. Suppression of irradiation hardening in nanoscale V/Ag multilayers. Acta Mater. 59, 6331-6340 (2011).

$6 \mathrm{Hu}, \mathrm{X}$. X., Xu D. H., Byun T. S. \& Wirth B. D. Modeling of irradiation hardening of iron after low-dose and low-temperature neutron irradiation. Modell. Simul. Mater. Sci. Eng. 22, 065002 (2014).

7 Ding, M. S. et al. Radiation-induced helium nanobubbles enhance ductility in submicron-sized single-crystalline copper. Nano Lett. 16, 4118-4124 (2016).

8 Polian, A. \& Grimsdit, M. Elastic Properties and Density of Helium up to $20 \mathrm{GPa}$. Europhys. Lett. 2, 849-855 (1986).

9 Landau, P., Guo, Q., Hosemann, P., Wang, Y. Q. \& Greer, J. R. Deformation of as-fabricated and helium implanted $100 \mathrm{~nm}$-diameter iron nano-pillars. Mater. Sci. Eng. A 612, 316-325 (2014).

10 Villacampa, I., Chen, J. C., Spätig, P., Seifert, H. P. \& Duval, F. Helium bubble evolution and hardening in 316L by post-implantation annealing. J. Nucl. Mater. 500, 389-402 (2018).

11 Plimpton, S. Fast parallel algorithms for short-range molecular dynamics. Journal of computational physics 117.1, 1-19 (1995).

12 Bonny, G., Grigorev, P. \& Terentyev, D. On the binding of nanometric hydrogen-helium clusters in tungsten. Journal of Physics: Condensed Matter 26.48, 485001 (2014).

13 Chen, Z., et al. Atomistic simulations of the effect of embedded hydrogen and helium on the tensile properties of monocrystalline and nanocrystalline tungsten. Journal of Nuclear Materials 481, 190-200 (2016).

14 Chen, W. Q., et al. Characterization of dose dependent mechanical properties in helium implanted tungsten. Journal of Nuclear Materials 509, 260-266 (2018).

15 Grigorev, P., et al. Interaction of hydrogen and helium with nanometric dislocation loops in tungsten assessed by atomistic calculations. Nuclear Instruments and Methods in Physics Research Section B: Beam Interactions with Materials and Atoms 393, 164-168 (2017). 
16 Jian, W-R., et al. Atomistic simulations of dynamics of an edge dislocation and its interaction with a void in copper: a comparative study. Modelling and Simulation in Materials Science and Engineering 28.4, 045004 (2020).

\section{Figure S1}
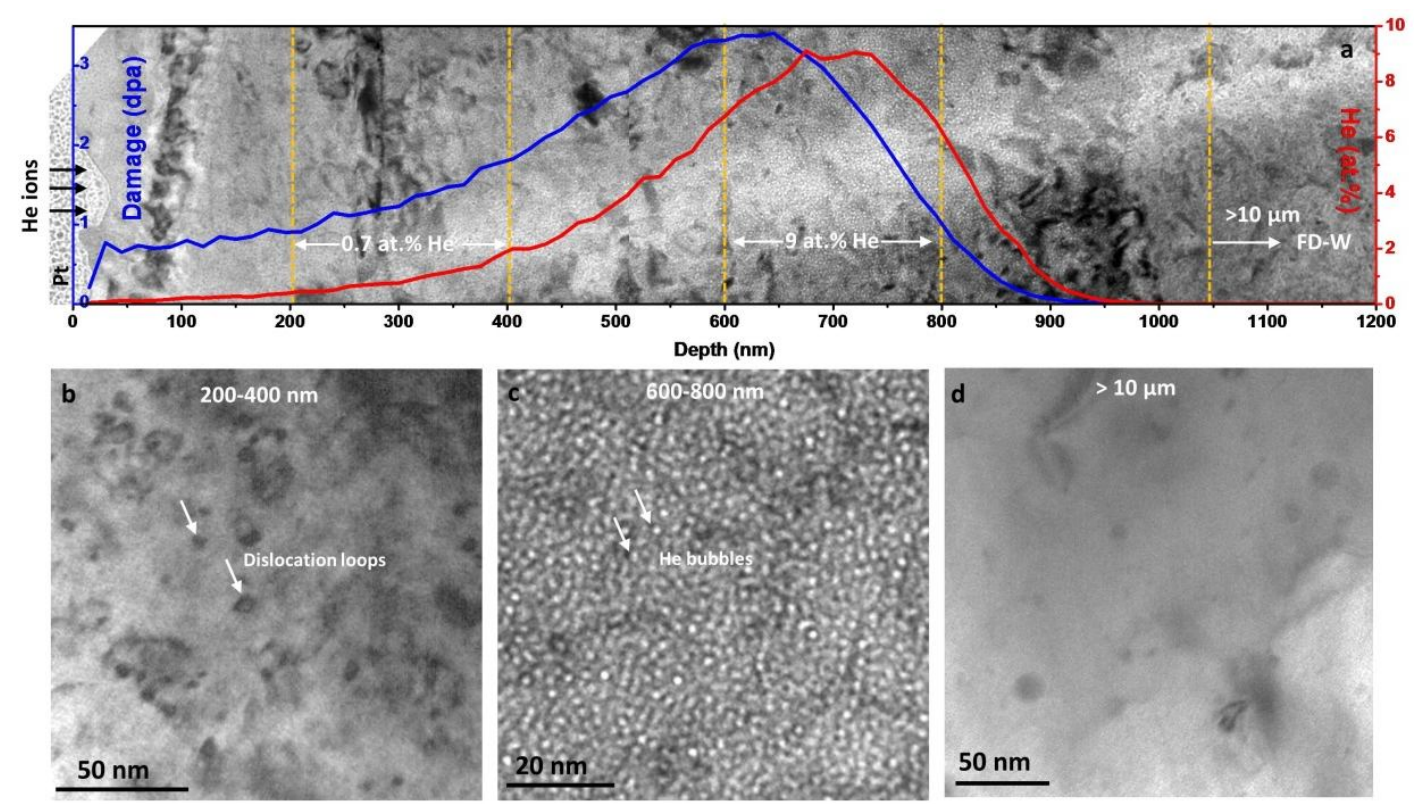

Figure S1. SRIM simulations and defects distribution in the He irradiated tungsten. (a) Defects distribution according to the SRIM calculation. (b) Dislocation loops in a low He concentration 0.7 at.\% He area at depths of 200-400 nm. (c) He bubbles in a high He concentration 9 at.\% He region at depths of 600-800 $\mathrm{nm}$. (d) Unirradiated areas far beyond a depth of $10 \mu \mathrm{m}$ without radiation defects.

\section{Figure S2}
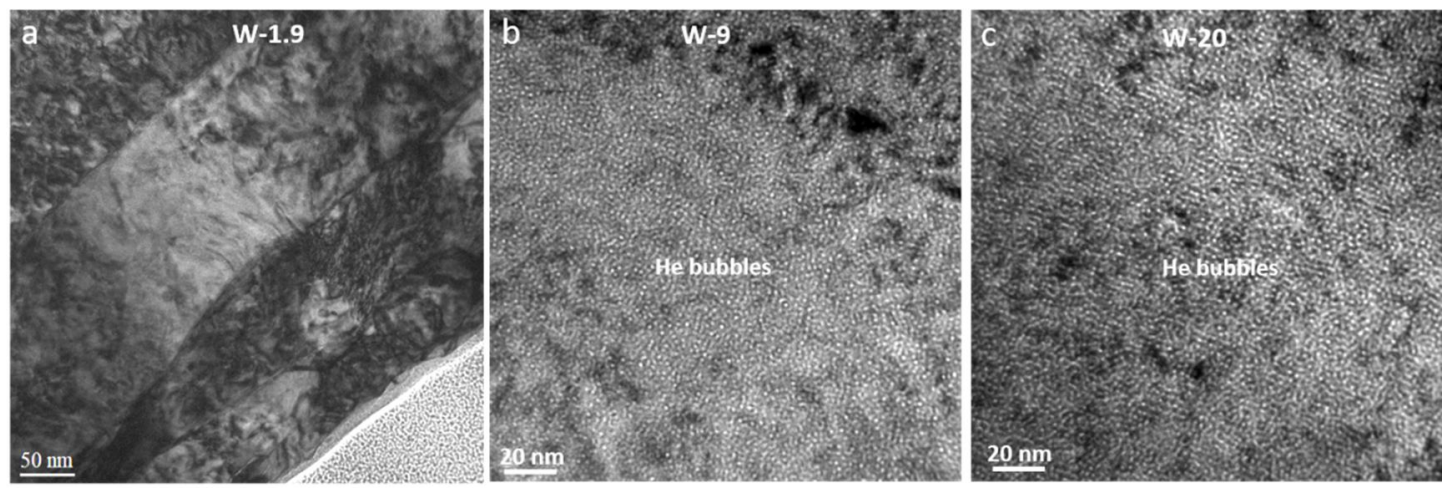

Figure 2. Typical images of He bubbles in W-1.9, W-9 and W-20 at the peak He concentration region. The images were taken with a defocus of $-1000 \mathrm{~nm}$. 
Figure S3

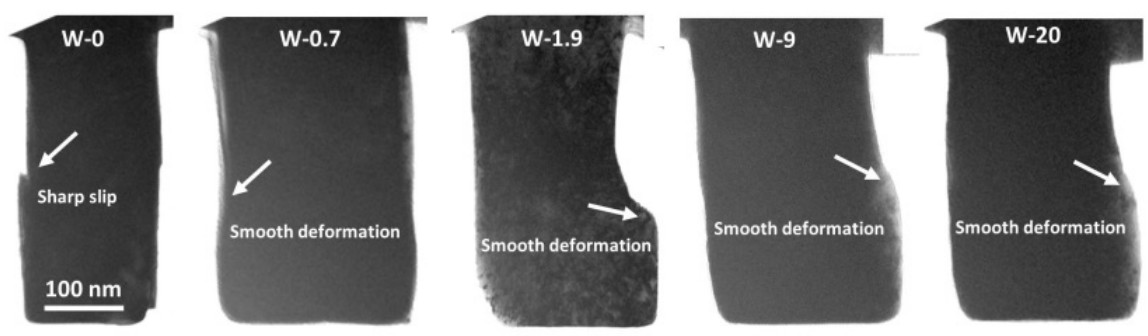

Figure S3. Morphologies of the compressed pillars. Morphologies of the W-0, W-0.7, W-1.9, W-9, and $\mathrm{W}-20$ pillars after compression.

Figure S4

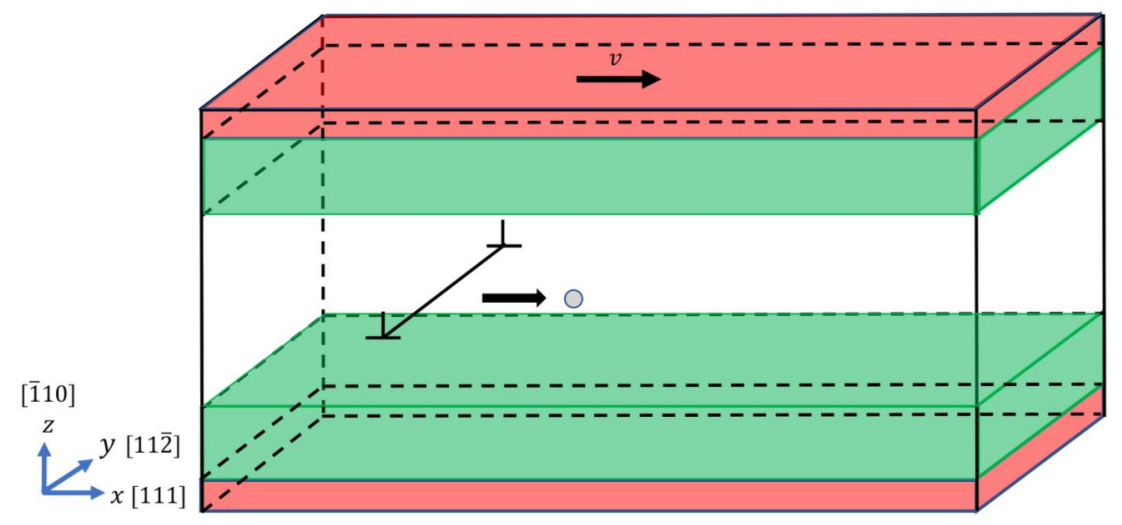

Figure S4. The schematic of the molecular dynamics model setup for simulating the stress-driven interaction of a dislocation and a He-vacancy complex in single crystal W. The red, green and non-colored regions represent the boundary, thermostat and non-thermostat regions. The gray sphere represents the He-vacancy complex.

Table S1. Properties of the He bubbles in the irradiated samples.

\begin{tabular}{|c|c|c|c|c|}
\hline Sample & W-0.7 & W-9 & W-1.9 & W-20 \\
\hline Fluence (ion $/ \mathrm{cm}^{2}$ ) & \multicolumn{2}{|c|}{$2 \times 10^{17}$} & \multicolumn{2}{|c|}{$5 \times 10^{17}$} \\
\hline Average bubble density $\left(\times 10^{22} / \mathrm{m}^{3}\right)$ & 1.4 & 150 & 1.6 & 220 \\
\hline Average bubble spacing (nm) & 205 & 21.32 & 192 & 17.47 \\
\hline Average bubble radius $(\mathrm{nm})$ & 0.85 & 0.72 & 0.85 & 0.75 \\
\hline Percentage of He atom in bubbles (\%) & 3.5 & 13 & 1.6 & 8.2 \\
\hline Ratio of He atoms to vacancies & 3.6 & 3.8 & 3.6 & 3.7 \\
\hline Pressure (GPa) & 6.3 & 7.5 & 6.3 & 7.2 \\
\hline
\end{tabular}

